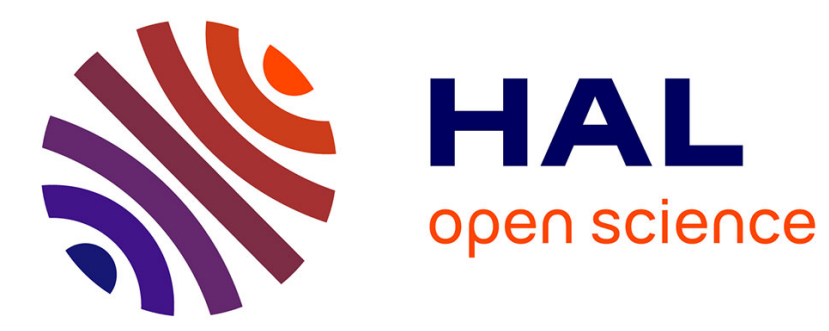

\title{
Reformulating Clancey's generic tasks for bridging both sides of dual reality
}

\author{
Walid Merrad, Alexis Heloir, Christophe Kolski
}

\section{To cite this version:}

Walid Merrad, Alexis Heloir, Christophe Kolski. Reformulating Clancey's generic tasks for bridging both sides of dual reality. 29ème conférence francophone sur l'Interaction Homme-Machine, AFIHM, Aug 2017, Poitiers, France. pp.137-146, 10.1145/3132129.3132135 . hal-01578490

\section{HAL Id: hal-01578490 \\ https://hal.science/hal-01578490}

Submitted on 29 Aug 2017

HAL is a multi-disciplinary open access archive for the deposit and dissemination of scientific research documents, whether they are published or not. The documents may come from teaching and research institutions in France or abroad, or from public or private research centers.
L'archive ouverte pluridisciplinaire HAL, est destinée au dépôt et à la diffusion de documents scientifiques de niveau recherche, publiés ou non, émanant des établissements d'enseignement et de recherche français ou étrangers, des laboratoires publics ou privés. 


\section{Reformulation des tâches génériques de Clancey pour relier les deux côtés de la réalité duale Reformulating Clancey's generic tasks for bridging both sides of dual reality}

\author{
Walid Merrad \\ LAMIH UMR CNRS 8201 \\ Univ. Valenciennes \\ 59313, Valenciennes, France \\ walid.merrad@etu. \\ univ-valenciennes.fr
}

\author{
Alexis Heloir \\ LAMIH UMR CNRS 8201 \\ DFKI / MMCI \\ Universität, Geb. E1.1, room 119 \\ 66123, Saarbrücken, Germany \\ alexis.heloir@dfki.de
}

\author{
Christophe Kolski \\ LAMIH UMR CNRS 8201 \\ Univ. Valenciennes \\ 59313, Valenciennes, France \\ christophe.kolski@ \\ univ-valenciennes.fr
}

\begin{abstract}
In many domains, the realization of an abstract model implies to cross the valley separating an abstract conceptualization and its actual completion in the physical world. In this paper, we propose a generic model based on Clancey's ontology of generic tasks and the dual reality paradigm. This model aims at bridging the real and the virtual sides of dual reality in the generic context of task/project realization. We describe existing implementations of the model, focusing on tangible tabletops - a medium particularly well suited to blend the real and the virtual worlds in a consistent tangible environment. Discussion, perspectives and conclusions are then presented.
\end{abstract}

\section{CCS CONCEPTS}

- Human-centered computing $\rightarrow \mathrm{HCI}$ theory, concepts and models; Interaction paradigms;

\section{KEYWORDS}

Dual reality, generic tasks, human-computer interaction.

\section{RÉSUMÉ}

Dans de nombreux domaines, la réalisation d'un modèle abstrait implique de traverser la vallée séparant une conceptualisation abstraite et son achèvement réel dans le monde physique. Dans cet article, nous proposons un modèle générique basé sur l'ontologie des tâches génériques de Clancey et le paradigme de la réalité duale. Ce nouveau modèle vise à relier les deux côtés, réel et virtuel, de la réalité duale dans le contexte générique de réalisation de tâches/projets. Nous décrivons des implémentations existantes du modèle, en nous concentrant sur des tables tangibles interactives -

(c) ACM, 2017. This is the author's version of the work. It is posted here by permission of ACM for your personal use. Not for redistribution.

The definitive version was published in Actes de la 29ème conférence francophone sur l'Interaction Homme- Machine, IHM '17, August 28-September 1, 2017, Poitiers, France

https://doi.org/10.1145/3132129.3132135 un moyen particulièrement adapté pour mélanger les mondes réel et virtuel dans un environnement tangible et cohérent. Une discussion, des perspectives et des conclusions sont ensuite présentées.

\section{MOTS-CLEFS}

Réalité duale, tâches génériques, interaction homme-machine.

\section{INTRODUCTION}

Novel HCI-mediated collaborative solutions are required to help users interact efficiently during the lifetime of complex projects, from their preliminary design phase occurring in a conceptual space to the final validation phase occurring in a physical space.

The conceptual space where experts elaborate blueprints and abstract models could be directly mapped onto the virtual world depicted in Lifton's Dual Reality model [20] whereas its counterpart, the physical space could be directly mapped onto the real world of the Dual Reality model.

In 1985, Clancey proposed an ontology of generic tasks capable of agnostically depicting the activity of users [9]. In this paper, we show that considering the Clancey model from a dual-reality perspective has the potential to enrich the set of generic tasks originally proposed by Clancey so they could fit tangible collaborative user interfaces, such as interactive tangible tabletops.

This paper is organized as follows : in Section 2 we discuss the concepts of dual reality, generic tasks and heuristic classification followed by a short synthesis connecting these concepts. In Section 3 we present and expose our proposed model for bridging the real and the virtual side of dual reality based on Clancey's work [9]. In Section 4 we describe some use cases using tangible user interfaces as proofs of concept. We discuss the model and its advantage, power and efficiency in Section 5. Finally, a concluding Section closes the paper by exposing future research directions.

\section{LITERATURE REVIEW ON DUAL REALITY AND GENERIC TASKS}

\subsection{Dual reality}

2.1.1 The dual reality concept. The concept of dual reality was proposed by Lifton in 2007 in his $\mathrm{PhD}$ thesis [20] as "an environment resulting from the interplay between the real world and the 
virtual world, as mediated by networks of sensors and actuators. While both worlds are complete unto themselves, they are also enriched by their ability to mutually reflect, influence, and merge into one another". He also writes that "sensor networks will turn the physical world into a palette, virtual worlds will provide the canvas on which the palette is used, and the mappings between the two are what will make their combination, dual reality, an art rather than an exact science. Of course, dual reality media will in no way replace other forms of media, but rather complement them". In [19] Lifton and Paradiso add to the above definition that both worlds are enhanced by the ability to mutually reflect, influence, and merge by means of sensor/actuator networks deeply embedded in everyday environments. In the same paper [19], the authors described a system demonstrating the Dual Reality paradigm : by a plug sensor node, it demonstrates the information flow from the real world to a virtual environment, implemented in the Second Life Online Virtual World ${ }^{1}$, where the data sensed from a real object (such as light, temperature, motion, sound and electrical current) influences the corresponding digital representation.

One of the late works in the field of dual reality is realized by Kahl [13], where he discussed the design of a management tool to monitor smart spaces in which sensors and actuators are installed and exchange data with the accordant services (such systems can be found in smart factories and urban management control stations). The author proposes a generic framework for controlling smart spaces. Additionally, this tool offers the possibility to add virtual services and to run simulations. He developed a two-component management dashboard. The real component, also called physical component, includes the sensors and actuators while the second component includes a virtual counterpart of the first one represented in three-dimensional model. These two worlds are connected and can influence each other according to the Dual Reality paradigm defined by Lifton in [20]. This means that the management dashboard aims at visualizing a real smart space in an interactive three-dimensional model in such a way that changes in the real world are immediately reflected in the virtual model and information from the virtual model can be transferred to the physical space.

In another paper of Kahl et Bürckert [14], the authors proposed an event-based communication infrastructure in order to enable interconnection between different services in an instrumented environment. The given description of the architecture matches the criteria of the Dual Reality Paradigm, defined by Lifton [20], as an interface between both worlds. Many application fields of the dual reality have been stated in several papers such as the management of warehouse and retail [15]. This work features a virtual dashboard offering a real time visualization of an actual supermarket in an interactive three-dimensional model. The virtual dashboard instantly reflects the changes which occured in the real world and information from the virtual world can be interpreted in the real world. Back et al. [4] designed a virtual factory which mirrors a real world chocolate factory ${ }^{2}$ located in San Francisco, USA. Sensor data is imported into the multi-user 3D environment from hundreds of sensors on the factory floor. The resulting virtual factory is used

1. http ://secondlife.com

2. TCHO VENTURES, INC. http ://www.tcho.com

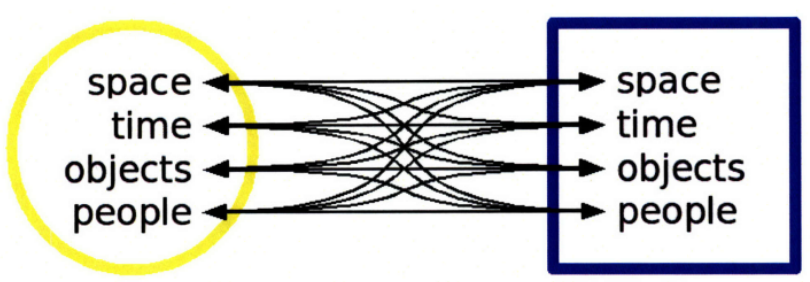

Figure 1: Fundamental mappings between the real (left) and the virtual (right) [20].

for simulation, visualization, and collaboration, using a set of interlinked, real-time layers of information. Their design support also industrial uses for mobile devices, such as cell phones and tablet computers. Back et al. cite that "through this mixture of mobile, social, mixed and virtual technologies, systems can be created for enhanced collaboration in industrial settings between physically remote people and places, such as factories in China with managers in the US" [4].

Another example consists of a model of a virtual apartment [21] in Dual Reality, the user can turn on the light of a lamp in the virtual environment. In the real counterpart, the light is turned on as well remotely by the software. Vice-versa if the user turns on the light in the real world, this is recognized by the software, which then turns on the light in the visualization [21].

The efficiency of dual reality, its performance and its task solution strategies are compared to those of virtual and real world in a study done by Raber et al. [21]. The results show that for certain tasks (like maximizing the profit) interacting with the virtual world yields to better results, whereas the best efficiency can be observed in a Dual Reality setup.

The difference between dual reality and mixed systems is that this last one merges both of physical and virtual components as one world [11], whereas the dual reality keeps the two -similar- worlds separated and ensure the link between them. Tangible interfaces (such as tangible tabletops) are considered as mixed systems [11] because of the interaction using tangible objects as input in the real world to manipulate digital information in the virtual world.

2.1.2 Establishing the mapping. There exist many challenges in designing the interaction and the mapping between the real and the virtual worlds. A direct mapping in both ways (from real to virtual and vice versa) is not always the most appropriate one : the sensor data streams collected from a real person may be better mapped to the virtual land the person's avatar rather than to the avatar itself [20] (see Fig. 1).

In the same paper [20], Lifton described another mapping strategy consisting of shaping the virtual world according to our subjective perceptions of the real world. The virtual world would be a reflection of reality distorted to match our mind's eye impressions as discerned by a network of sensors. An example, one could recognize the buildings on a virtual campus which could change in size according to the number of inhabitants and virtual corridors which could widen or lengthen according to their actual throughput.

The work of [13] [4] and [21] demonstrates some implementations in a dual reality setup. Thus the interconnection between the 
two worlds depends on the context of the application and is not within a well structured framework and/or architecture. In addition, the interconnection between the two worlds is made instinctively in each case separately. We propose a principled generic model fitting many application contexts built around an ontology of composable generic tasks.

\subsection{Generic tasks}

2.2.1 The concept of generic tasks. The need for generic tasks evolves from the fact that the level of abstraction of much work in Knowledge-Based Systems (e.g rules, frames, logic) is too low to provide a rich vocabulary for knowledge and control. Chandrasekaran [7] provided an overview of a framework called the Generic Task approach that proposes that knowledge systems should be built out of building blocks, each of which is appropriate for a basic type of problem solving. Each generic task uses forms of knowledge and control strategies that are characteristic to it, and are generally conceptually closer to domain knowledge. He follows next in the same paper [7] that the abstract specification of a generic task is :

- The function of the task. What type of problem does it solve? What is the nature of the information that it takes as input, and produces as output?

- The representation and organization of knowledge. What are the primitive terms in which the forms of knowledge needed for the task can be represented? How should knowledge be organized and structured for that task?

- The control strategy. What control strategy (inference strategy) can be applied to the knowledge to accomplish the function of the generic task?

In another paper of Chandrasekaran [8], it is stated that each generic task relies on forms of knowledge and control strategies that are characteristic to it, and are in general conceptually closer to domain knowledge. In [6] authors mention that each generic task is characterized by information about the following :

- The type of problem (the type of input and the type of output). What is the generic task used for?

- The representation of knowledge. How should knowledge be organized and structured to accomplish the function of the generic task? In particular, what are the types of concepts that are involved in the generic task? What concepts are the input and output about? How is knowledge organized in term of concepts?

- The inference strategy (process, problem solving, control regime). What inference strategy can be applied to the knowledge to accomplish the function of the generic task? How does the inference strategy operates on concepts?

Clancey has also worked on generic tasks and operations, beside knowledge engineering. We find in his famous paper [9] a generic model for operations (tasks) that we can do to or with a system. Fig. 2 and Fig. 3 summarize hierarchically these generic operations. Operations are grouped in terms of those that construct a system and those that interpret a system, corresponding to what is generally called synthesis and analysis.

Clancey describes that the terms between brackets are common synonyms of the generic operations (in capital letters). He also explains in [9] that INTERPRET operations concern a working system in some environment. In particular, IDENTIFY is different from DESIGN in that it requires taking $\mathrm{I} / \mathrm{O}$ behavior and mapping it onto a system.

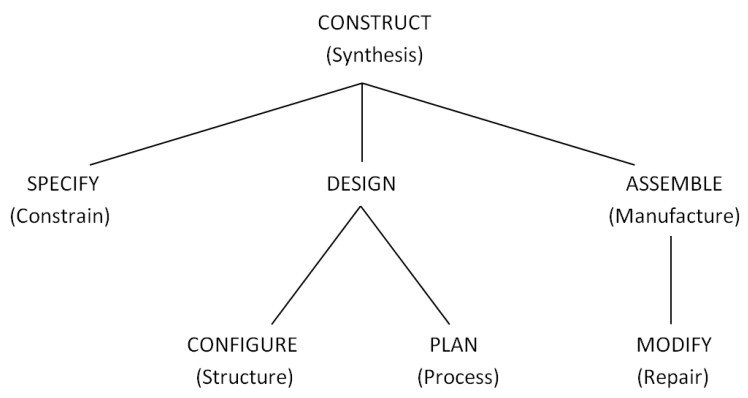

Figure 2: Generic operations for synthesizing a system.

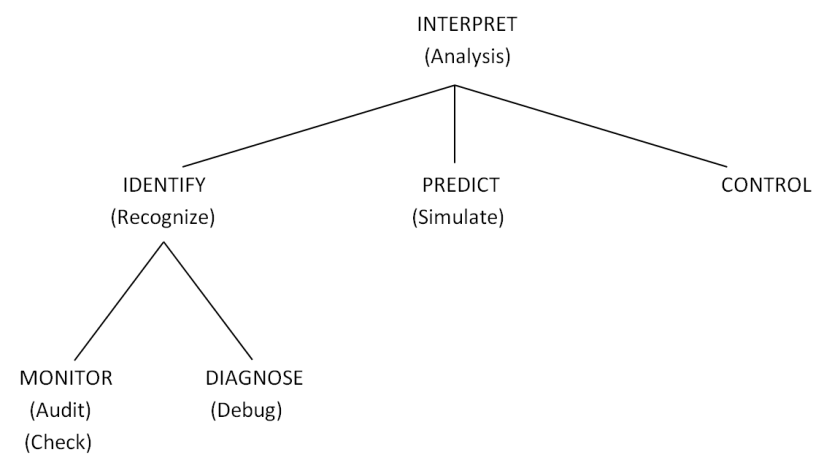

Figure 3: Generic operations for analyzing a system.

Whilst PREDICT is the inverse, taking a known system and describing output behavior for given inputs. Moreover, Simulate is a specific method for making predictions, suggesting that there is a computational model of the system, complete at some level of detail. For the CONTROL, not often associated with heuristic programs, takes a known system and determines inputs to generate prescribed outputs [25]. Thus, these three operations, IDENTIFY, PREDICT and CONTROL, logically cover the possibilities of problems in which one factor of the set input, output, system is unknown.

Further explanations are given in [9], when the author notes that MONITOR and DIAGNOSE presuppose a pre-existing system design against which the behavior of an actual, running system is compared. Thus, one identifies the system with respect to its deviation from a standard. In the case of MONITOR, one detects discrepancies in behavior (or simply characterizes the current state of the system). In the case of DIAGNOSE, one explains monitored behavior in terms of discrepancies between the actual (inferred) design and the standard system.

The Design is taken to be the general operation that embraces both a characterization of structure (CONFIGURATION) and process (PLANNING). DESIGN is conceptual, it describes a system in terms of spatial and temporal interactions of components. Therefore, The idea of "executing a plan" is moved to the more general 
term ASSEMBLE, meaning the physical construction of a system [9]. Also from the same reference, SPECIFY refers to the separable operation of constraining a system description, generally in terms of interactions with other systems and actual realization in the world (resources affecting components). Of course, in practice design difficulties may require modifying the specification, just as assembly may constrain design (commonly called "design for manufacturing").

2.2.2 Generic tasks and $\mathrm{HCl}$. The researches on Human-Computer Interaction and cognitive sciences keep rising year after another. Meanwhile, the importance of task modeling and analysis has also become more important than ever, such as in computer science and in human automation.

The interest of doing tasks analysis can be seen in three main points : update an exiting system to get a new one, create a new system from many existing systems and create a new system from scratch [23]. Kolski in his book [16] and Diaper et al. in [10] described several human tasks modelling and analysis methods. Some of them are based on software engineering approaches, while some others are based on teamwork collaboration and ergonomics

G.A. Boy in his book [5] dedicated a chapter for concepts and tools for designers, where he discussed the human-centered design (HCD) and the task/activity distinction towards a system. He proposes to combine HCD and technology-centered engineering to make human-systems integration. He says that we should focus more on human tasks while designing a system since the beginning of the process, and also to distinguish between a task (what is prescribed on user requirements) and an activity (what is effectively performed). This difference has place often between the practice (physical world) and the theory (virtual ideal world). Therefore, to fill this gap between these two worlds, using generic tasks can guarantee a standardization of user tasks or interactions towards the system, resulting in rationalization of different kind of interactions and more stability of the system. It would also allow to avoid any unexpected behaviour from the user that may question the efficiency of the system.

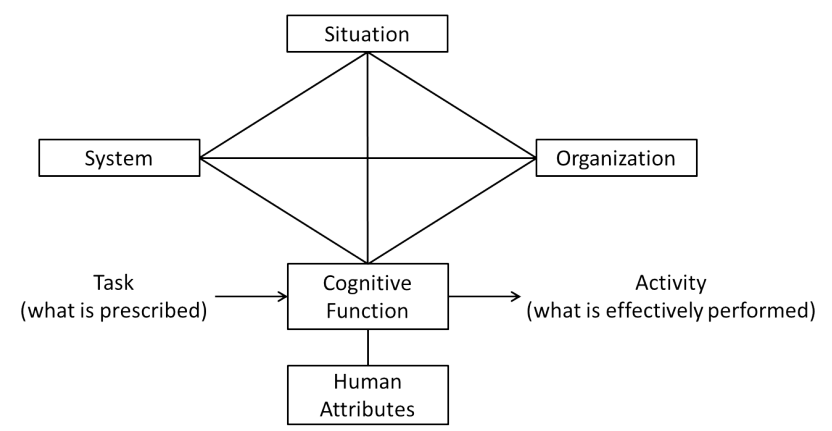

Figure 4: Transformation of a task into an activity [5].

Fig. 4 shows a cognitive function as a transformation of a task into an activity, influenced by human attributes (e.g., fatigue, motivation, competence), the current situation (i.e., the state of the world around), the organization (i.e., the various actors involved in the execution of the task), and the system itself [5].

\subsection{Summary}

We have seen this far that the dual reality consists of two connected worlds, one real and one virtual, that interact between them and influence each other using a network of sensors and actuators. We have also been through some works such as the generic framework tool of Kahl [13], the virtual factory [4] and the virtual apartment [21]. Afterwards, we described the concept of generic tasks, detailed the heuristic classification of Clancey [9] and gave links between the generic tasks and HCI. We try to combine all of these concepts in our proposal to obtain a model that ensures the bridging of both dual reality sides.

\section{OUR PROPOSAL}

Our work is based on the fusion of the dual reality paradigm and the generic tasks concepts of Clancey [9] which we exposed in the previous Section. We propose this generic model shown in Fig. 5 for mapping between the two worlds and ensure a generic manner of interaction from and towards real and virtual worlds.

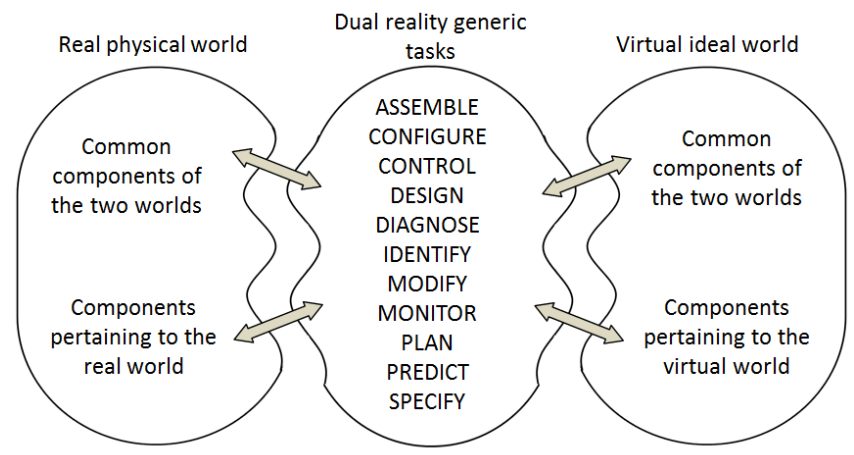

Figure 5: Generic bridging model between the real and virtual worlds.

Our proposal allows to generically model the user activities, as well as to bridge the both sides of dual reality. We note that the interaction is bidirectional (as defined in the dual reality paradigm) and each element from one world, whether it is a common component or not, can influence and be influenced by the same world or the other world components, be they common or not. The standard components of the two worlds are elements that we find in both of them, with the same properties. For instance, object states, machine properties, and user inputs are all common between the two worlds. While for components of one world, they are elements that can be found in one world but not in the other. However, they can be represented by similar elements. For example, a person is a real physical component of the real world that can be represented by an avatar in the virtual world, which cannot exist in the real world (see Fig. 5).

Moreover, the bidirectional arrows show that an object may interact with another object from the same world (and actually the same family), using one or many generic tasks. In this case, the interaction will be reproduced in the other world between the corresponding elements of the corresponding generic task(s). The curved zigzags between the three most important parts of the model Fig. 5) indicate 
that there are no clear limits. Thus, merging or bridging both sides of the dual reality is similar to making these boundaries transparent for the users and providing a human-computer interface that ensures the same environment, in the real or virtual world, while using a system.

The sensors and actuators networks will ensure the communication : sensing, sending and actioning, regardless of the used protocols, between the two worlds. Information (instructions, user data, etc.) can circulate and be exchanged based on the model for any possible combination of components as described previously. The exchange is done according to one or many generic tasks and cannot take any form else.

Any interaction can be constructed and described using the generic tasks listed in the middle part of Fig. 5. In fact, according to Clancey [9] we can combine some of those generic elementary tasks to get a sequence of operations to solve a given problem. The two commonly occurring sequences are :

- The construction cycle : SPECIFY + DESIGN $\{+$ ASSEMBLE $\}$.

- The maintenance cycle : $\{$ MONITOR + PREDICT + $\}$ DIAGNOSE + MODIFY.

Clancey notes in [9] that "heuristic classification is well-suited for problems of interpretation involving a system that is known to the problem solver", and this includes intelligent interactive systems. For example, the Kinect of Microsoft enables a broad range of interaction with old and new applications [26]. For instance, using body gestures (whole body, hands, head, etc.) to calibrate the Kinect for a game, the user has to go through multiple primitive and combined generic tasks, we mention for example and not limited to :

- CONTROL : moving the cursor to select an item in the screen,

$-\{$ MONITOR + PREDICT $\}$ : also known as test, user checks each time he or she makes an interaction if the result is convenient or not,

- MODIFY : when the user moves an object on the screen using the cursor (and his/her hand),

- IDENTIFY : the user can identify from a set of known device positions which one suits him/her well for a given game.

Also, the construction cycle can be approached while setting up a game (via Kinect or usual joystick). The user has to SPECIFY some criteria, such as how many players, DESIGN the game like choosing places, environments and players characteristics. Finally, the user ASSEMBLE the system (the game) after validation.

According to Clancey, the maintenance cycle is the familiar pattern of medical programs, such as MYCIN [22]. The test consists of repeatedly observing system behavior on the input selected to verify output predictions.

In the following Section, we will demonstrate the implementation of our model based on several examples.

\section{IMPLEMENTATION OF THE MODEL ON SEVERAL REPRESENTATIVE SYSTEMS}

Numerous examples from daily life can be given in this context, in different domains. Since our work is related to HCI, we provide some examples based on interactive tangible tabletops as described next in Section 4.1, Section 4.2 and Section 4.5. We deconstruct five representative systems, three of them are previous work we conducted (see Section 4.1, Section 4.2 and Section 4.5). In each case, the model exhibits both a physical (real) and a virtual facet. We rely on Clancey's ontology to identify the tasks belonging to the virtual domain, the tasks belonging to the physical domain and the ones laying at the interface between the two domains.

\subsection{Cooking Ideas}

In [18], authors proposed a tabletop equipped with RFID technology and a distributed display (computer screen) to suggest additional information (see Fig. 6). This tabletop allows interactions with tangible objects (real ingredients) to take place and suggests recipes to the user. While in the screen, the user can see different information about the ingredients and what's missing, the recipe, etc. Thus, to provide all functionalities, the application has to be run on two different supports (the screen and the tabletop), displaying different but complementary content.

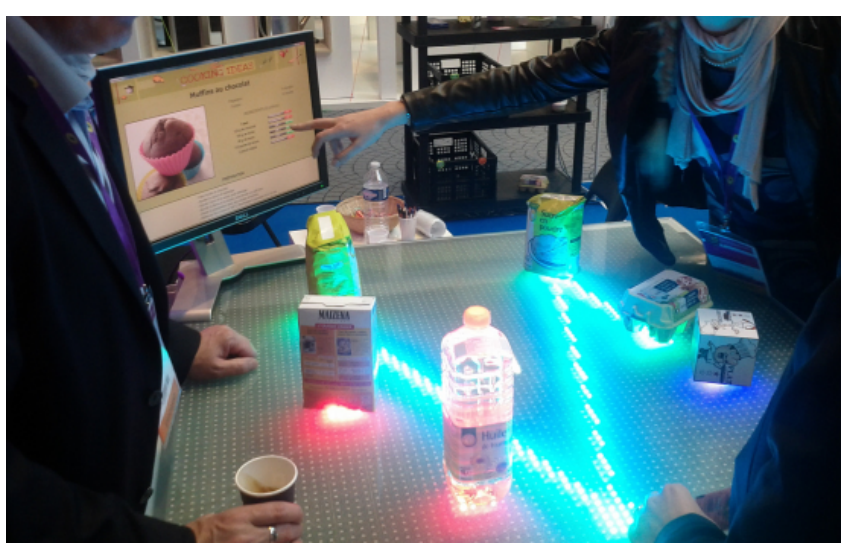

Figure 6: The cooking ideas distributed on two surfaces [18].

In this case, as shown in Fig. 7, the recipe is considered as the ideal model, and the meal is the real (physical) side of the dual reality, while the tabletop and the application are found to be in between these two. Also, the user set constraints at the beginning of the session when specifying which type of recipe to be used (main dish, dessert, etc.). The kind of recipe is a standard component of the two worlds and can influence the recipe to be made from the real or the virtual world. 


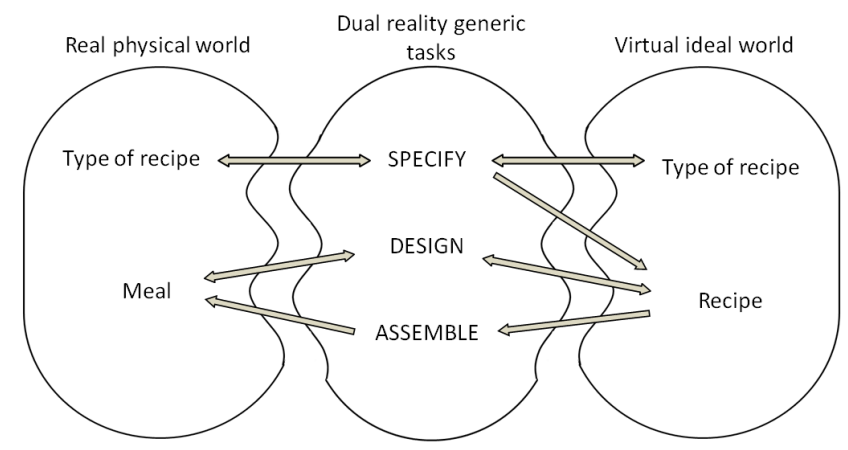

Figure 7: Cooking ideas application implemented under our model.

DESIGN is defined in [12] and used by Clancey in his paper [9] as "configuring objects under constraints". Therefore, preparing a recipe can be considered as a task of design.

\subsection{Colors recognition and learning}

In [17] authors show how they used the TangiSense interactive tabletop in recognition and learning of colors in a nursery school. The experiment consisted of objects that have lost their colors and children (aged from 2 to 6 years) must place them in the right colored frame on the tabletop. A "magician" (tangible object) is then used to launch the checking procedure and announces to the child whether he/she has made any errors or has given correct answers (see Fig. 8).

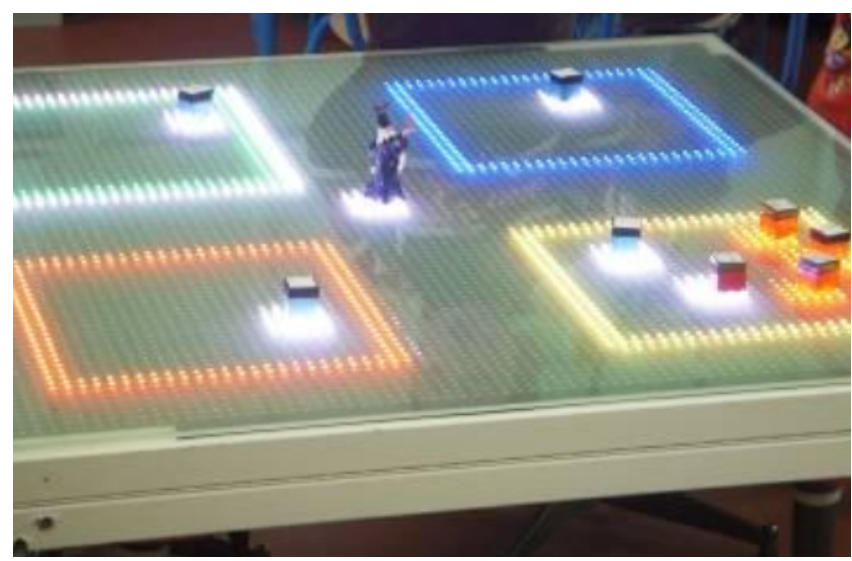

Figure 8: The TangiSense interactive table with the "Recognition and learning of colors" application [17].

Thus, the performed interaction consists first of IDENTIFYING objects' colors. Once the objects placed in their frames, here comes the sequence of MONITOR and PREDICT to test (check) if the objects are well positioned. In the case of a wrong answer, the user needs to replace the object(s) in another (other) color frame(s), and this is a task of MODIFICATION. This interaction is described in Fig. 9.

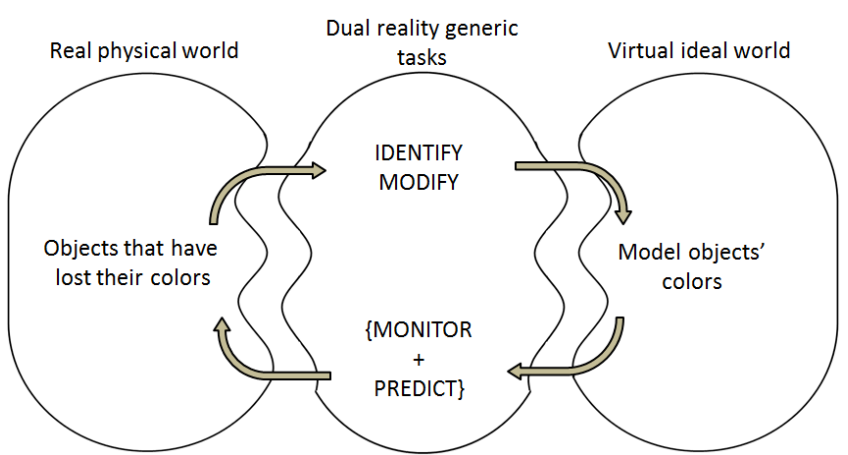

Figure 9: Colors recognition and learning interactions according to our model.

In Fig. 9, we notice the loop of tasks that we described previously (identify the color, place the object then check). The user can start whether from the real or the virtual world, perform a task and switch the interaction to the other world. The loop ends when all answers are correct.

\subsection{Construction site}

Experts in constructions (architects, civil engineers, etc.) can use our model to check, in every step, that what was planned and designed matches with what is realized in reality by the workmen in the field. The real physical world is the construction site, and the virtual abstract world is the architectural plan, while the mediation between the two can be the digital model. Fig. 10 shows a routine check on a construction site and a 3D digital model of the building.

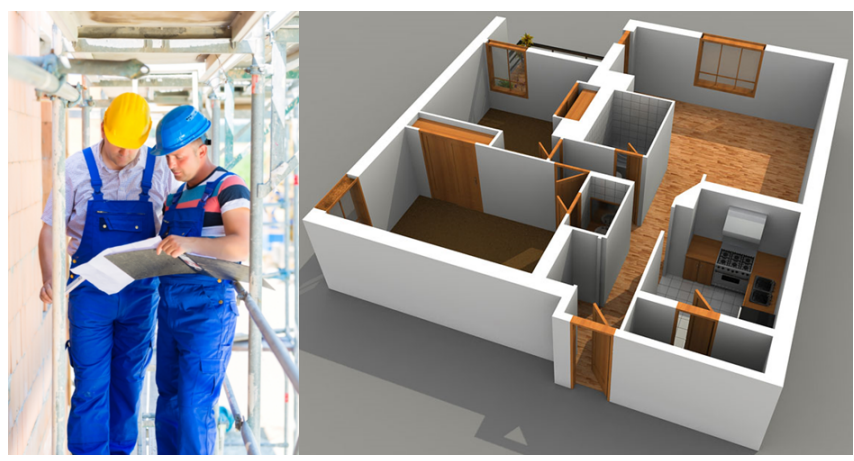

Figure 10: A civil engineer controlling the construction site (left) [1] and a 3D digital model of the building (right) [2]

Fig. 11 illustrates possible interactions in this example. The building and its infrastructure are done (ASSEMBLE) according to the architectural plan, with possible changes (MODIFY) during the realization, and new laws can influence the architectural scheme. Also, experts and engineers check (MONITOR) that work standards and rules are respected in the construction site and plan for the realization of the project. We note that the schedule, for example, may be influenced in both directions. 


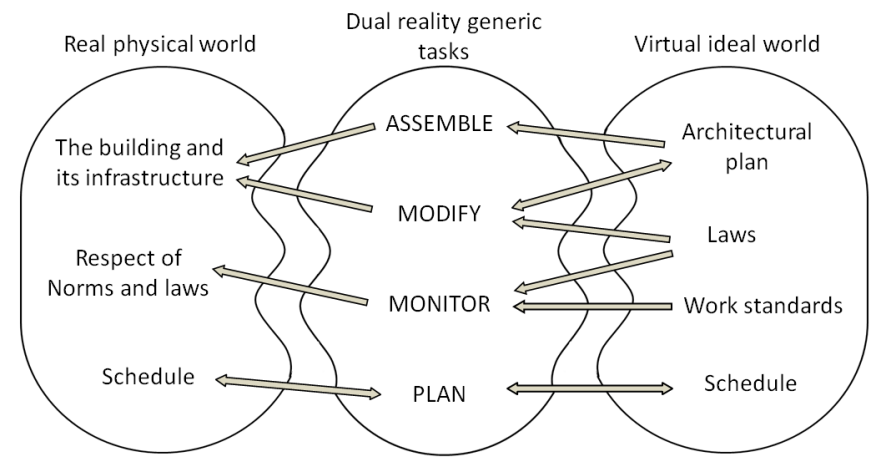

Figure 11: A construction project interactions according to our model.

\subsection{The virtual factory}

The virtual plant concept described in [4] is full of use cases that can be used to demonstrate our proposal. Fig. 12(a) shows the plant in the real world while Fig. 12(b) shows the plant and an avatar in the virtual world.

(a)

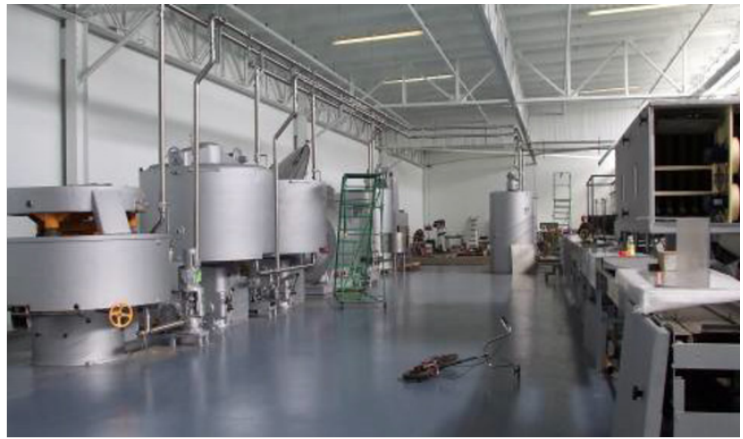

(b)

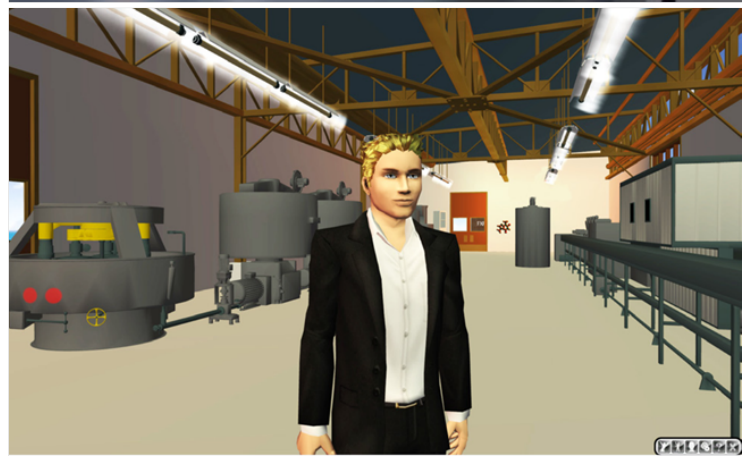

Figure 12: (a) the TCHO factory floor under construction, (b) an avatar in the Multiverse Virtual Factory [4].

In fact, a controller in the supervision room may control and change production machines configuration (CONTROL); a workman can apply the same thing in the production room. Also, the managers can PLAN for the manufacturing process and maintenance using the sensed data from the virtual or the real world. They can CONFIGURE production and storage process as well, MONITOR the system behavior and PREDICT system breakdowns. Fig. 13 shows these interactions modelled under our proposed model.

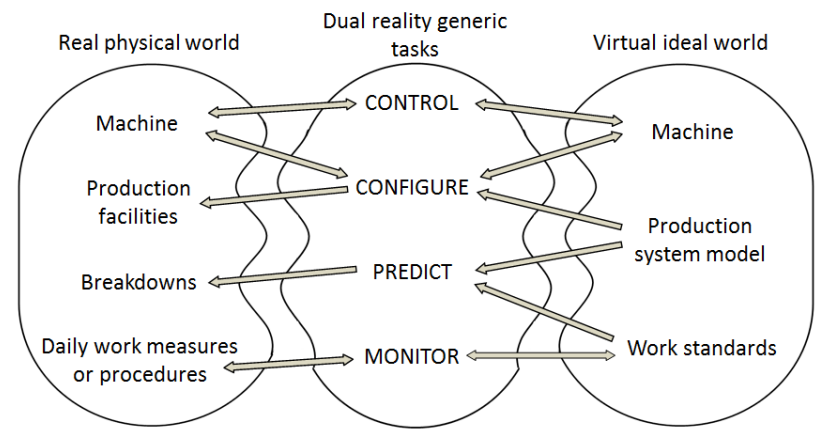

Figure 13: Implementation of the virtual factory interactions.

We note that some interactions are bidirectional, such as configuring or controlling a machine. While some others are unidirectional, showing a one-way interaction. For instance, system breakdowns can be predicted from the production system model and the work standards of the factory, but these last two abstract concepts cannot be affected by a system breakdown.

\subsection{Music on tangible tabletops}

The TangiSense Music Application [3] shown in Fig. 15 allows users to compose (DESIGN and ASSEMBLE) and edit (MODIFY) music sequences on different rhythms and using various instruments. Users can set (SPECIFY) the rhythm of the music and use a generic sound object to inspire sounds that do not exist. Fig. 16 illustrates the interactions of this system. The system can be described according to the model proposed, with two different worlds (real and virtual) and generic tasks bridging them.

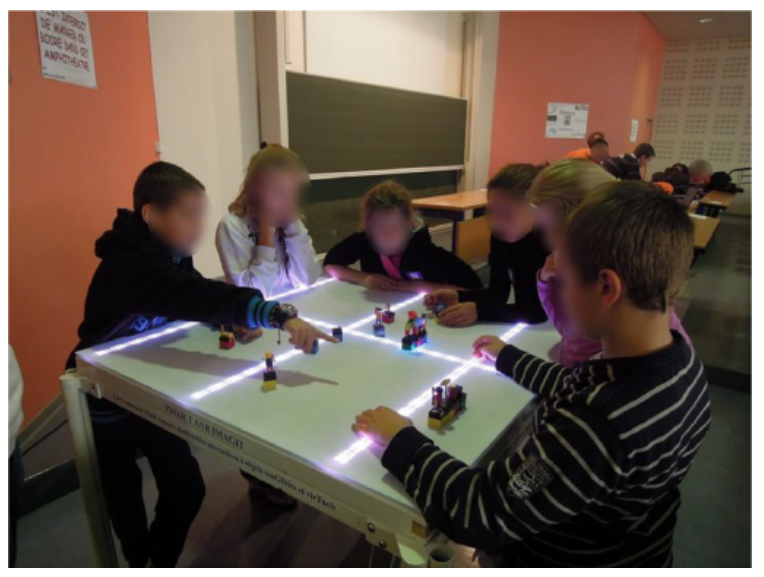

Figure 15: Some pupils using the music creation application. 


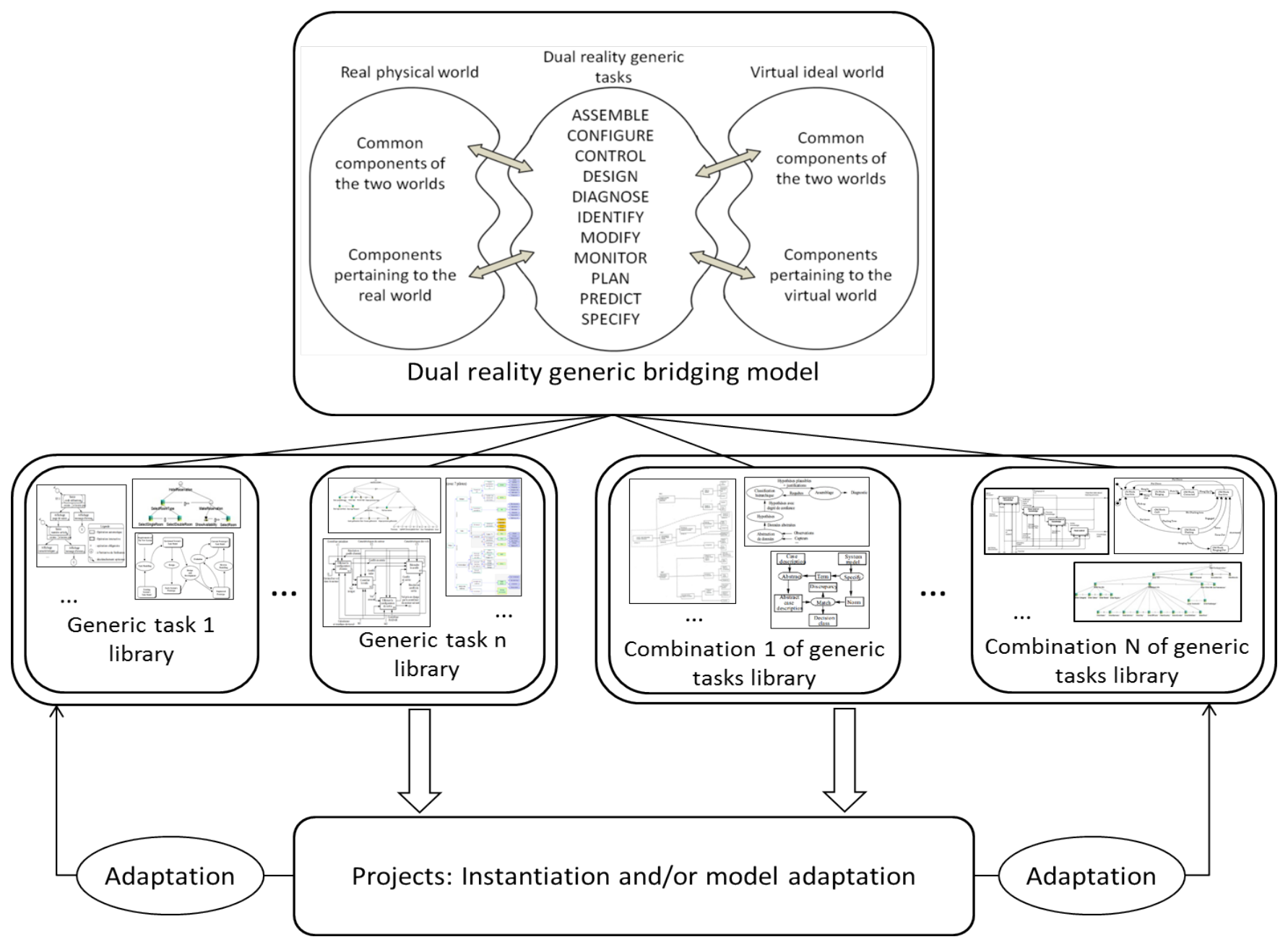

Figure 14: Possible extensions of the proposed model.

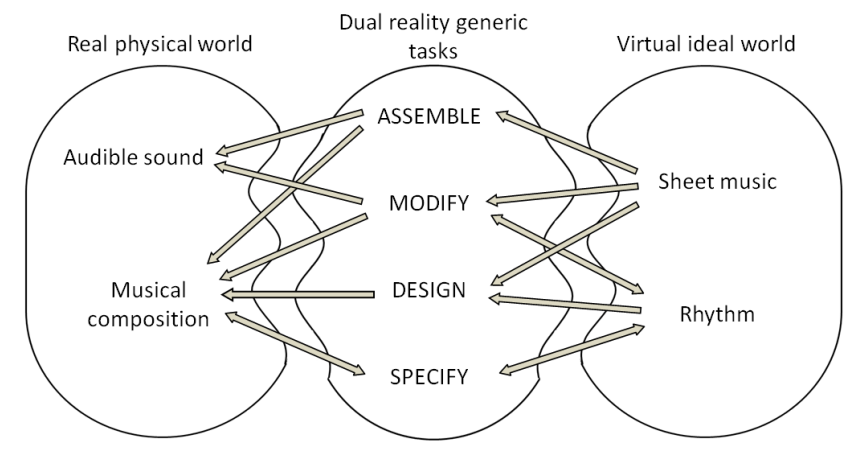

Figure 16: Implementation of the music application interactions.

In this example, the music score and the rhythm are considered to be the ideal models, and the user may compose (DESIGN, ASSEMBLE and MODIFY) them to form an audible sound (one simple musical note) and a musical composition (consisting of several attached simple musical notes). Meanwhile, the audible sound and the musical composition are what the user hears after a performance in the real world. The user can set (SPECIFY) the rhythm of the music he/she composes, and we note that the rhythm can be influenced by the user from the real world or by the Sheet music (model) from the virtual world. The operation of MODIFY consists of changing the structure of the system, in this case, editing the sequence of musical notes. SPECIFY refers to constraining the system, in this case, the white lines on the tabletop move across the whole surface of the tabletop and users can set their speed so that the music notes will be played under this (rhythm) constraint.

\section{DISCUSSION}

In this paper, we have drafted a universal model for representing complex activities like problem solving, exploration, or design. In this model, a bidirectional mapping connects a set of atomic tasks scattered over three adjacent conceptual spaces : the abstract space, the dual space, and the physical space. In the abstract space, virtual 
ideal models and abstract aspects are represented. In the physical space, real and concrete aspects from the physical world are to be found, whereas in the dual space, the generic tasks represent the interface that bridges between the physical and the abstract spaces.

Using Clancey's ontology of generic tasks, we deconstructed 5 applications in different domains. We could show that these various applications fit well into the generic model we proposed.

The model described in this paper is not final yet : we are currently working on a formalization based on Clancey's principled approach. We will conduct this formalization effort iteratively, enriching and validating the model at each step using demonstrators. Ultimately, this effort would lead to the specification of generic components accounting for the dual nature of many use cases as well as the tangible nature of the interaction. Therefore, it is too premature to challenge Clancey's reference model, which has proven its resilience and genericity.

So far, no dual reality framework or architectures have been proposed. However, similar researches have been conducted : for instance, the visual monitoring and management tool for smart environments by Kahl [13] and "Architecture to Enable Dual Reality for Smart Environments" by Kahl and Bürckert [14], but no bridging model exists. Also, what exists allows certain functionalities and depends on the context of usage. Thus the bridging of the real and virtual worlds in a dual reality context does not submit to any model, and it is done case by case severally.

Fig. 14 illustrates possible extensions of the model we proposed. Each generic task model can be detailed in a dedicated library for many usages, depending on the context. Different combinations of tasks can also be modeled and stored in libraries dedicated to this. Models of various libraries can then be used, and combined as required, in different projects. The use of a task model in a project includes its instantiation and its adaptation to the needs. Other task models may be created, adapted and added to libraries to be reused again later. In their turn, these last may be combined with other tasks and get used in projects.

\section{CONCLUSION}

The concept of generic tasks combined with the dual reality paradigm that we presented allows to connect and bridge the real and virtual worlds, in a way that interactions in one world are duplicated in the other world, via the reformulated model of Clancey [9] we proposed. In this work, we have seen some works on dual reality and generic tasks, as well as the link between them. We have also presented our model for bridging both sides of dual reality, inspired from the generic tasks used in Clancey's heuristic classification and from the dual reality paradigm. We provided some implementation examples in real life systems to show the usage of our model.

The perspectives of this work include detailing the flows that bridge the two sides of dual reality (real and virtual), but also detailing the flow of each generic task of the model using one or several task models and implementing the proposed model in dual reality applications, particularly on the tangible tabletop TangiSense equipped with RFID capture technology. Another important research perspective is to reuse and extend the formalization (formal logic) used by Clancey in his paper in the following research work. Ongoing work is about implementing this model in a dual reality application for crisis management, which consists of controlling robots on the field using the TangiSense interactive tabletop and mobile tanks (dynamic tangible objects). Any action in one world (be it real or virtual) will be duplicated and performed in the other world to maintain the similarity between these two worlds. Based on [24], we also intend to produce "user-defined gestures" for tangible interaction in a dual reality environment.

\section{ACKNOWLEDGEMENTS}

The Fig. 15 comes from a work session with Sophie Lepreux and Yoann Lebrun. This research was financed by the French Ministry of Education, Higher Education and Research, in mediation by the Hauts-de-France Region. The authors would like to thank the institution funding this research and for the doctoral grant.

\section{RÉFÉRENCES}

[1] 2017. Achats d'intérim : chantier RH au sein de Bouygues Construction. http://www.decision-achats.fr/Thematique/process-outils-1037/Breves/ Achats-interim-chantier-sein-Bouygues-Construction-306978.htm. (2017). [Online; accessed 14-April-2017].

[2] 2017. Outsource 3D CAD Modeling. http://www.outsource3dcadmodeling.com/ images/3dcadsamples/3d-floor-plan-1.jpg. (2017). [Online ; accessed 14-April2017].

[3] Daniel Arfib, Jehan-Julien Filatriau, and Loïc Kessous. 2009. Prototyping musical experiments for Tangisense, a tangible and traceable table. Gouyon, F.; Barbosa, A. ; Serra, X, Proceedings of 6th Sound and Music Computing Conference 2009, pp. 247-252 (2009)

[4] Maribeth Back, Don Kimber, Eleanor Rieffel, Anthony Dunnigan, Bee Liew, Sagar Gattepally, Jonathan Foote, Jun Shingu, and James Vaughan. 2010. The virtual chocolate factory : mixed reality industrial collaboration and control. In Proceedings of the 18th ACM international conference on Multimedia. ACM, 1505-1506.

[5] Guy André Boy. 2016. Tangible Interactive Systems. Springer.

[6] David C Brown and Balakrishnan Chandrasekaran. 2014. Design problem solving : knowledge structures and control strategies. Morgan Kaufmann.

[7] Balakrishnan Chandrasekaran. 1987. Towards a Functional Architecture for Intelligence Based on Generic Information Processing Tasks.. In IFCAI, Vol. 87. 1183-1192.

[8] Balakrishnan Chandrasekaran. 1988. Generic tasks as building blocks for knowledge-based systems : the diagnosis and routine design examples. The Knowledge Engineering Review 3, 03 (1988), 183-210.

[9] William J Clancey. 1985. Heuristic classification. Artificial intelligence 27, 3 (1985), 289-350.

[10] Dan Diaper and Neville Stanton. 2003. The handbook of task analysis for humancomputer interaction. CRC Press.

[11] Emmanuel Dubois. 2009. Conception, Implémentation et Evaluation des Systèmes Interactifs Mixtes : une Approche basée Modèles et Centrée sur l'Interaction. HDR Thesis, Toulouse. (2009).

[12] Frederick Hayes-Roth, Donald Waterman, and Douglas Lenat. 1984. Building expert systems. Addison-Wesley, Reading, MA.

[13] Gerrit Kahl. 2013. A visual monitoring and management tool for smart environments. In Proceedings of the companion publication of the 2013 international conference on Intelligent user interfaces. ACM, 93-94.

[14] Gerrit Kahl and Christian Bürckert. 2012. Architecture to enable dual reality for smart environments. In Intelligent Environments (IE), 2012 8th International Conference on. IEEE, 42-49.

[15] Gerrit Kahl, Stefan Warwas, Pascal Liedtke, Lübomira Spassova, and Boris Brandherm. 2011. Management dashboard in a retail scenario. In Workshop on Location Awareness in Dual and Mixed Reality. International Conference on Intelligent User Interfaces (IUI-11). 22-25.

[16] Christophe Kolski. 1997. Interfaces Homme-machine : application aux systèmes industriels complexes. Hermès.

[17] Sébastien Kubicki, Sophie Lepreux, and Christophe Kolski. 2011. Evaluation of an interactive table with tangible objects : Application with children in a classroom. In Proceedings 2nd workshop on child computer interaction" UI Technologies and Educational Pedagogy", at CHI'2011, Vancouver, Canada, may.

[18] Yoann Lebrun, Sophie Lepreux, Sylvain Haudegond, Christophe Kolski, and René Mandiau. 2014. Management of distributed RFID surfaces : a cooking assistant for ambient computing in kitchen. The 5th International Conference on Ambient 
Systems, Networks and Technologies, ANT-2014 (fune 2-5, 2014, Hasselt, Belgium), Procedia Computer Science 32 (2014), 21-28.

[19] Joshua Lifton and Joseph A Paradiso. 2009. Dual reality : Merging the real and virtual. In International Conference on Facets of Virtual Environments. Springer $12-28$.

[20] Joshua Harlan Lifton. 2007. Dual reality : an emerging medium. Ph.D. Dissertation Massachusetts Institute of Technology.

[21] Frederic Raber, Antonio Krüger, and Gerrit Kahl. 2015. The Comparison of Performance, Efficiency, and Task Solution Strategies in Real, Virtual and Dual Reality Environments. In Human-Computer Interaction - INTERACT 2015. IFIP Conference on Human-Computer Interaction (INTERACT-15), 15th IFIP TC 13 International Conference, September 14-18, Bamberg, Germany, J. Abascal, S. Barbosa, M. Fetter T. Gross, P. Palanque, and M. Winckler (Eds.). Springer.

[22] Edward Shortliffe. 2012. Computer-based medical consultations : MYCIN. Vol. 2. Elsevier.
[23] R. B. Stammers, M. S. Carey, and J. A. Astley. 1990. Task analysis. In J. R. Wilson and E. N. Corlett (eds.) Evaluation of human work. A Practical Ergonomics Methodology, Taylor and Francis. (1990).

[24] Consuelo Valdes, Diana Eastman, Casey Grote, Shantanu Thatte, Orit Shaer, Ali Mazalek, Brygg Ullmer, and Miriam K. Konkel. 2014. Exploring the design space of gestural interaction with active tokens through user-defined gestures. In Proceedings of the SIGCHI Conference on Human Factors in Computing Systems. ACM, 4107-4116.

[25] Venkateswararao Vemuri. 2014. Modeling of complex systems : an introduction. Academic Press.

[26] Zhengyou Zhang. 2012. Microsoft kinect sensor and its effect. IEEE multimedia 19, 2 (2012), 4-10. 